\title{
COVIMPACT: PANDEMIA COVID-19 NOS ESTUDANTES DO ENSINO SUPERIOR DA SAÚDE
}

COVimpact: COVID-19 pandemic in students of higher health education

COVimpact: pandemia de COVID-19 entre estudiantes de educación superior en salud

\author{
António Ferreira ${ }^{*}$, Fernanda Príncipe ${ }^{* *}$, Henrique Pereira $^{* * *}$, Isabel Oliveira ${ }^{* * *}$, Liliana Mota ${ }^{* * * *}$
}

\section{RESUMO}

Enquadramento: no contexto de emergência de saúde pública internacional por SARS-CoV-2, a suspensão das atividades letivas presenciais criou uma disrupção ao regular funcionamento das instituições de ensino superior com impacte na comunidade académica. Objetivo: avaliar o impacte da pandemia COVID-19 no percurso académico e de vida dos estudantes e sua satisfação com as medidas adotadas. Metodologia: estudo descritivo, exploratório. Recolha de dados por questionário online, com questões centradas nas condições para o ensino à distância, nas vivências dos estudantes e no impacte e satisfação com o Plano de Contingência. A amostra não probabilística e de conveniência. Análise estatística dos dados de acordo com a natureza das variáveis. Resultados: participaram 264 estudantes com idade média de $28,2 \pm 10,2$ anos. No geral, referem muito boas condições em casa para concretização do ensino à distância $(\bar{X}=4,4 \pm$ $0,67,2-5)$. A maioria $(79,2 \%, n=209)$ refere ter participado nas atividades não presenciais após a ativação do Plano de Contingência. Da análise do impacte verificam-se níveis de adequação muito elevados das plataformas educativas. Constata-se também, uma elevada satisfação com a informação fornecida. Conclusão: as medidas adotadas foram adequadas tendo contribuído para reduzir o impacte do COVID-19 no processo de ensino, aprendizagem e avaliação dos estudantes.

Palavras-chave: pandemia; ensino superior; estudantes

${ }^{*} \mathrm{MsC}$, Professor Adjunto na Escola Superior de Saúde Norte da Cruz Vermelha Portuguesa, Doutorando em Enfermagem na Universidade Católica Portuguesa. https://orcid.org/0000-0001-5008-3746

**PhD, Professora Coordenadora na Escola Superior de Saúde Norte da Cruz Vermelha Portuguesa/CINTESIS.

https://orcid.org/0000-0002-1142-3258

${ }^{* * *}$ PhD, Professor Coordenador na Escola Superior de Saúde Norte da Cruz Vermelha Portuguesa.

https://orcid.org/0000-0002-0362-2513

${ }^{* * * *}$ RN, Professora Adjunta. Especialista em Enfermagem de reabilitação. https://orcid.org/0000-0001-6627-3907

${ }^{* * * * *} \mathrm{PhD}$, Professora Adjunta na Escola Superior de Saúde Norte da Cruz Vermelha Portuguesa/CINTESIS.

https://orcid.org/0000-0003-3357-7984
Como Referenciar:

Ferreira, A., Príncipe, F., Pereira, H., Oliveira, I., \& Mota, L. (2020). Covimpact: pandemia convid-19 nos estudantes do ensino superior da saúde. Revista de Investigação \& Inovação em Saúde, 3(1), 7-16. doi:10.37914/riis.v3i1.80

Recebido para publicação em: 20/05/2020 Aceite para publicação em 09-06-2020

\section{ABSTRACT}

Background: in the context of an international public health emergency due to SARS-CoV-2, the suspension of presence teaching activities created a disruption to regular functioning of higher education institutions with impact on the academic community. Objective: to assess the impact of the COVID-19 pandemic on the academic and life path of students and their satisfaction with the measures adopted. Methodology: descriptive, exploratory study. Data collection by online questionnaire, centered on the conditions for distance learning, on the students' experiences and on the impact and satisfaction with the Contingency Plan. A non-probabilistic and convenient sample was used. Statistical analysis of the data according to the nature of the variables. Results: 264 students participated with average age of $28.2 \pm 10.2$ years. In general, they report very good conditions at home to implement distance learning $(\bar{X}=4,4 \pm 0,67,2-5)$. The majority $(79.2 \%$, $n=209$ ) reported having participated in non-presence activities after activating the Contingency Plan. The impact analysis shows very high levels adequacy of educational platforms. There is also a high level of satisfaction with the information provided. Conclusion: the measures adopted were adequate and contributed to reduce the impact of COVID-19 on the teaching, learning and assessment process of students.

Keywords: pandemics; higher education; students

\section{RESUMEN}

Marco contextual: el contexto de emergencia internacional de salud pública por SARS-CoV-2, la suspensión de actividades presenciales de enseñanza creó una interrupción en el funcionamiento de las instituciones de educación superior con impacto en la comunidad académica. Objetivo: evaluar el impacto de la pandemia COVID-19 en la trayectoria académica y de vida de los estudiantes, y su satisfacción con las medidas adoptadas. Metodología: estudio descriptivo, exploratorio. Recolección de datos por cuestionario online, centrado en las condiciones para el aprendizaje, las experiencias, el impacto y la satisfacción con el Plan de Contingencia. La muestra es no probabilística de conveniencia. Análisis estadístico de los datos según la naturaleza de las variables. Resultados: 264 estudiantes participaron con edad media de $28.2 \pm 10.2$ años. En general, informan muy buenas condiciones en el hogar para implementar el aprendizaje a distancia $(\bar{X}=4,4 \pm 0,67,2-5)$. La mayoría $(79.2 \%, n=209)$ ha participado en actividades no presenciales. El análisis de impacto muestra niveles muy altos de adecuación de las plataformas educativas. También hay un alto nivel de satisfacción con la información proporcionada. Conclusión: las medidas adoptadas fueron adecuadas y contribuyeron a reducir el impacto de COVID-19 en el proceso de enseñanza, aprendizaje y evaluación de los estudiantes.

Palabras clave: pandemia; enseñanza superior; estudiantes 


\section{INTRODUÇÃO}

Num contexto de emergência de saúde pública internacional e com a chegada dos primeiros casos de infeção por SARS-CoV-2 a Portugal, a 13 de março o Governo português decretou um conjunto de medidas excecionais e temporárias, visando conter a propagação da infeção, sendo uma delas a medida de suspensão de atividades letivas presenciais nas instituições de ensino superior (Decreto-Lei n.ำ10$\mathrm{A} / 2020$, de 13 de março).

Atenta ao contexto epidemiológico vivido a Escola Superior de Saúde Norte da Cruz Vermelha Portuguesa (ESSNorteCVP), mesmo antes de ter sido decretada pelo Governo a suspensão das atividades letivas presenciais, havia já, no âmbito do seu Plano de Contingência COVID-19, encetado diferentes estratégias destinadas a proteger a comunidade académica, incluindo a suspensão da atividade letiva presencial a 9 de março de 2020 e assegurar a prossecução dos percursos de ensino aprendizagem dos seus estudantes (Escola Superior de Saúde Norte da Cruz Vermelha Portuguesa, 2020).

No entanto, e associada a todas as outras medidas de confinamento impostas aos cidadãos portugueses, a suspensão das atividades letivas presenciais criou uma disrupção ao regular funcionamento das instituições de ensino superior com expectável impacte em toda a comunidade académica, especialmente nos estudantes.

Face a este novo contexto, importa avaliar o impacte da pandemia COVID-19 no percurso académico e de vida dos estudantes e sua satisfação com as medidas adotadas pela ESSNorteCVP. Os resultados deste estudo permitirão igualmente aferir a adequação das medidas até agora implementadas pela
ESSNorteCVP, no sentido de minimizar os constrangimentos e limitações vivenciados pelos estudantes na consecução do seu percurso académico.

\section{ENQUADRAMENTO/FUNDAMENTAÇÃO TEÓRICA}

No final de 2019 foram identificados casos de pneumonia atípica provocados pelo SARS-CoV2, que rapidamente se propagou a nível mundial. A COVID19, como depois foi denominada, foi declarada como emergência de saúde pública internacional em janeiro de 2020, sendo que em março a Organização Mundial de Saúde viria a caraterizar esta infeção como pandémica (World Health Organization, 2020). Desde cedo as medidas que se mostraram mais eficazes na interrupção da transmissão local da infeção foram as medidas de confinamento social, restrições à movimentação de pessoas, distanciamento social e consciencialização das populações para as medidas de proteção individual (Cheng, Jian \& Liu, 2020; Zhang, Litvinova, Wang, Wang, Deng, \& Chen, X.... Yut, H. 2020), levando a que muitos países tenham decretado, em larga escala, a suspensão de muita da atividade económica e social, incluindo a cessação de toda a atividade letiva presencial.

O encerramento de estabelecimentos de ensino por todo o mundo para conter a disseminação da COVID19 provocou uma disrupção nos processos de ensino e aprendizagem de milhões de estudantes. A Organização para a Cultura, Ciência e Educação das Nações Unidas estima que cerca de $70 \%$ da comunidade estudantil mundial tenho sido afetada por esta medida, representando mais de 1198 milhões de estudantes em todos os níveis de ensino 
(United Nations Educational, Scientific and Cultural Organization, 2020a).

Os custos económicos e sociais resultantes da aplicação das medidas de confinamento social são muito elevados, para além de criarem constrangimentos e desafios adicionais às instituições de ensino e toda a comunidade académica aos mais diversos níveis. Estes incluem a interrupção dos processos de ensino aprendizagem, privando os estudantes de oportunidades de crescimento e desenvolvimento; a maior probabilidade de abandono dos estudos; a necessidade de criação, manutenção e aumento de resposta no ensino à distância que representou um desafio enorme em termos técnicos e humanos para a concretização da mudança da sala de aula, à grande escala e num tão curto espaço de tempo, para o contexto virtual; o desafio de medir e validar os resultados da aprendizagem; o isolamento social forçado, entre outros (United Nations Educational, Scientific and Cultural Organization, 2020b). O isolamento social pode ter efeitos devastadores incluindo sintomas de stresse pós-traumático, confusão e raiva, podendo os seus efeitos persistir a longo prazo (Brooks, Webster, Smith, Woodland, Wessely \& Greenberg, 2020).

À escala global existem diversas iniciativas de partilha de boas práticas e de recursos que permitem minimizar o impacte nos processos de ensino, aprendizagem e avaliação dos estudantes, assim como, na adoção de medidas de suporte ao teletrabalho de docentes/investigadores e outros colaboradores dentro da comunidade académica (International Association os Universities, 2020). Alguns países já passaram situações semelhantes causadas por catástrofes como o furacão Katrina ou o vírus SARS, embora a situação atual assuma contornos nunca antes vivenciados pelas sociedades atuais. Em todas estas calamidades o ensino à distância apresentou-se como a solução para dar continuidade às atividades letivas. Fizeram-se investimentos em plataformas de elearning, formação docente e dotaram-se os estudantes de meios tecnológicos (Sobral, 2020).

A COVID-19 não pode ser vista como tendo consequências positivas, mas pode ser encarada como uma oportunidade para tomar consciência que as catástrofes acontecem e que as instituições de ensino superior têm de estar preparadas para situações limite, garantindo que mesmo em situações tão adversas como as que agora vivenciam, estejam preparadas para assegurar que o processo de ensino e aprendizagem se mantém de forma ininterrupta durante este período.

United Nations Educational, Scientific and Cultural Organization (2020c) faz várias recomendações no sentido de potenciar as estratégias de aprendizagem à distância, das quais se destacam: a proteção da privacidade e segurança dos dados alocados nos espaços virtuais; a priorização de soluções em resposta aos desafios psicossociais previamente às questões do ensino; desenvolver regras de ensino a distância e monitorizar o processo de aprendizagem dos estudantes; e criar comunidades e melhorar a conexão entre as pessoas.

Deste contexto emerge a questão de investigação: qual o impacte da pandemia COVID-19 no percurso académico e vida dos estudantes da ESSNorteCVP em resposta à implementação do Plano de Contingência? 


\section{METODOLOGIA}

Estudo descritivo, exploratório desenvolvido na Escola Superior de Saúde Norte da Cruz Vermelha Portuguesa. A recolha de dados foi efetuada com recurso ao questionário online "COVImpact: questionário de avaliação do impacte COVID-19 versão estudantes". A construção do instrumento de recolha de dados resultou de uma reunião de peritos no qual participou o Presidente do Conselho de Direção, Presidente do Conselho Técnico-Científico, Presidente do Conselho Pedagógico, Presidente do Conselho para Avaliação da Qualidade e Direção de Departamento de Área de Ensino, tendo por base as diretivas do Ministério Ciência, Tecnologia e Ensino Superior, Direção Geral da Saúde e o Plano de Contingência COVID-19 da ESSNorteCVP.

O questionário é composto por questões centradas na caraterização da amostra, condições para o ensino à distância, as vivências dos estudantes durante o distanciamento físico, o impacte e satisfação com o Plano de Contingência COVID-19 no processo de aprendizagem, assim como sugestões de melhoria.

As variáveis do instrumento foram operacionalizadas numa escala de Likert que varia entre campos semânticos, 1 e 5 (Discordo totalmente - Concordo totalmente).

A amostra do estudo é não probabilística e de conveniência, constituída por estudantes dos cursos pré e pós-graduado em funcionamento. Participaram no estudo 264 estudantes, apresentando uma taxa de retorno de 52\%. A recolha de dados decorreu em maio de 2020 e foram incluídos no estudo todos os estudantes que frequentam o ano letivo 2019/2020 da ESSNorteCVP. Foi realizada análise estatística descritiva com recurso ao programa Statistical Package for the Social Sciences (SPSS) versão 23.0. A participação no estudo foi voluntária, garantido o anonimato e dada a possibilidade aos participantes de desistirem do estudo sem qualquer prejuízo. A realização do estudo foi autorizada pelo Conselho de Direção da ESSNorteCVP.

\section{RESULTADOS}

Os participantes do estudo têm em média $28,2 \pm 10,2$ anos variando entre 18 e 59 anos. Na sua maioria são do género feminino $(86 \%, n=227)$. Verifica-se que, por nível de ensino, $16,7 \%(n=44)$ dos estudantes frequentam os cursos de pós-graduação, 13,3\% $(n=35)$ frequentam os cursos de pós-licenciatura de especialização em enfermagem, $64,8 \% \quad(n=171)$ frequentam o 1 o ciclo de estudos do curso de enfermagem, $3,4 \%(n=9)$ frequentam o 1 o ciclo de estudos do curso de acupuntura, 0,4\% (n=1) frequentam o 1 o ciclo de estudos do curso de osteopatia e $1,5 \% \quad(n=4)$ frequentam o CTESP termalismo e bem-estar. À data da implementação do plano de contingência COVID-19 da ESSNorteCVP $63,6 \%(n=168)$ dos estudantes estavam a frequentar o processo de ensino aprendizagem com aulas de tipologia teórica, teórico-prática e prática e laboratorial, os restantes $(36,4 \%, n=96)$ frequentavam o processo de ensino clínico/estágio. Em média coabitam com os estudantes $3,4 \pm 1,28$ pessoas variando entre 0 e 12 pessoas. Na sua habitação encontram-se em média 1,45 $\pm 0,93$ pessoas em teletrabalho/tele escola variando entre 
0 e 6 pessoas. No geral os participantes referem muito boas condições em casa para concretização do ensino à distância $(\overline{\mathrm{X}}=4,4 \pm 0,67,2-5)$, com disponibilidade de computador ou tablet para acesso às atividades. Todavia, $0,8 \%(n=2)$ assinalam ter apenas acesso às atividades letivas com recurso a telemóvel.

Durante o isolamento em casa na sua maioria os estudantes revelam que têm sentido um estado de maior stresse e ansiedade (42,8\%, $n=113)$, têm sentido estados de maior apatia e desânimo (25\%, $\mathrm{n}=66)$, não têm sentido qualquer alteração do comportamento em relação ao que vivenciavam antes do isolamento em 22,8\% (n=60) dos estudantes, $3,8 \%(n=10)$ sentem-se mais agitados e $5,7 \%(n=15)$ referem sentir outra situação que não especificam.

Com a suspensão da atividade presencial os estudantes na sua maioria $(23,5 \%, n=62)$ referem só terem saído de casa para ir ao jardim ou quintal, $18,9 \%(n=50)$ referem ter saído à rua (ou espaço comum de um condomínio) duas a três vezes por

Tabela 1

Descritiva do impacte do plano de contingência COVID-19

\begin{tabular}{|c|c|c|c|c|c|}
\hline & $\mathrm{N}$ & Mínimo & Máximo & Média & Desvio Padrão \\
\hline Adequação das Plataformas educativas & 262 & 2 & 5 & 4,26 & 0,76 \\
\hline Adequação das medidas/estratégias & 261 & 1 & 5 & 4,21 & 0,72 \\
\hline Manutenção do ensino à distância & 262 & 1 & 5 & 4,11 & 0,98 \\
\hline Interação desenvolvida entre docentes e estudantes & 260 & 1 & 5 & 3,83 & 0,84 \\
\hline Adequação das Provas de avaliação & 186 & 2 & 5 & 3,82 & 0,79 \\
\hline Impacto no seu bem-estar físico e/ou mental & 264 & 1 & 5 & 3,64 & 0,99 \\
\hline Envolvimento com as atividades à distância & 261 & 1 & 5 & 3,29 & 1,05 \\
\hline
\end{tabular}

semana, $12,9 \%(n=34)$ raramente saiu à rua (ou espaço comum de um condomínio), 8\% (n=34) ficou sempre em casa, $6,4 \% \quad(n=17)$ tem saído à rua afastando-se do local onde reside mais que uma vez por semana, 4,5\% $(n=12)$ tem saído à rua (ou espaço comum de um condomínio) todos os dias para espairecer e $25,8 \%(n=68)$ referem outra situação que não especificam.

$\mathrm{Na}$ sua maioria $(79,2 \%, \mathrm{n}=209)$ os estudantes referem ter participado nas atividades não ativação do Plano de Contingência COVID-19, 11\% $(n=29)$ refere não ter participado e 9,8 \% $(n=26)$ refere não ter conhecimento dessas atividades.

Da análise do impacte do plano de contingência COVID-19 verifica-se que a adequação das plataformas educativas para o ensino não presencial demonstrou níveis de adequação muito elevados. A questão que apresentou níveis médios mais baixos foi o envolvimento dos estudantes com as atividades desenvolvidas à distância (Tabela 1). presenciais promovidas pela ESSNorteCVP após a 
Do ponto de vista da satisfação com a implementação do plano de contingência COVID-19 constata-se elevada satisfação com a informação fornecida pela ESSNorteCVP perante o cenário atual de COVID-19. A questão que apresentou níveis médios de satisfação mais baixos foi o envolvimento da Associação Académica, de acordo com os seus desígnios estatutários, em prol das necessidades dos estudantes que resultassem da pandemia (Tabela 2).

Tabela 2

Descritiva da satisfação com a implementação do plano de contingência COVID-19

\begin{tabular}{|c|c|c|c|c|c|}
\hline & $\mathrm{N}$ & Mínimo & Máximo & Média & Desvio Padrão \\
\hline Satisfação com a informação fornecida & 259 & 1 & 5 & 4,18 & 0,72 \\
\hline Satisfação com o ensino à distância & 257 & 1 & 5 & 3,73 & 0,89 \\
\hline Satisfação com o ensino à distância no ensino clínico & 169 & 1 & 5 & 3,32 & 0,95 \\
\hline $\begin{array}{l}\text { Satisfação com o envolvimento/ação/representação } \\
\text { da Associação Académica }\end{array}$ & 239 & 1 & 5 & 3,15 & 1,17 \\
\hline Satisfação Global & 261 & 1 & 5 & 3,84 & 0,91 \\
\hline
\end{tabular}

No que se refere às medidas adotadas pela ESSNorteCVP que mais contribuíram para reduzir o impacte do COVID-19 no processo de ensino os estudantes referiram a rápida adaptação da escola na criação de ambientes favoráveis ao ensino à distância síncrono com recurso a plataformas educativas, e ainda, a referência e o acompanhamento constante dos docentes que revelaram elevada disponibilidade.

Como medidas complementares que deveriam ser adotadas decididamente na redução do impacte do COVID-19 no processo de ensino de uma forma geral os estudantes reiteram e enaltecem as medidas adotadas pela escola, salientando a relevância do envio para a comunidade académica dos estudos baseados na evidência acerca da temática COVID pela Unidade Investigação e Desenvolvimento.

Todavia, os estudantes dos cursos de licenciatura sugerem a colocação antecipada do material de apoio à aula na plataforma Moodle $^{\circledR}$ e atividades mais dinâmicas nas aulas que decorrem com recurso às plataformas educativas. Os estudantes do ensino pós-graduado (pós-graduações e cursos de póslicenciatura de especialização em enfermagem) referem que os cursos deveriam ter sido suspensos pelo facto de verem o seu percurso formativo interrompido e não se terem inscrito em cursos online. Consideram ainda relevante a disponibilização de workshops, formação à distância síncrona e estudos de caso com doentes em ambiente virtual que pudessem ocupar horas dos estágios, interrompidos pelas instituições de saúde. Os estudantes de formação pré e pós-graduado consideram que poder-se-ia otimizar os fluxos de comunicação bidirecionalmente.

Como oportunidades de melhoria para o Sistema Interno de Garantia e Gestão da Qualidade os estudantes sugerem a implementação de medidas 
para avaliação da saúde mental e bem-estar dos estudantes, uniformização dos modelos de provas de avaliação via Moodle ${ }^{\circledR}$, manutenção do ensino à distância por forma a evitar nova vaga COVID, melhorar a gestão da carga horária nos horários escolares, promoção de debates sobre o desenvolvimento de competências, aulas de apoio aos trabalhos de grupo na formação pós-graduada e mais esclarecimentos sobre proteção de dados. Os estudantes trabalhadores consideram que a medida adotada pela escola, com a implementação do ensino à distância síncrono, lhes permitiu poupança económica e de tempo.

Os estudantes finalistas sugerem que thes seja permitida a realização da cerimónia de encerramento do curso com a presença dos seus familiares.

\section{DISCUSSÃO}

A pandemia COVID-19 obrigou a implementação de um plano de contingência que os estudantes consideraram adequado, o que minimizou o impacte no seu percurso académico e de vida, repercutindose em níveis elevados de satisfação com as medidas adotadas pela ESSNorteCVP. Verificam-se níveis muito elevados de satisfação com a adequação das plataformas educativas adotadas para o ensino não presencial. O ensino à distância apresenta-se como a solução para dar continuidade às atividades letivas, como já se verificou em anteriores calamidades de saúde à escala global (Sobral, 2020). De acordo com Camacho, Fuly, Santos \& Menezes (2020) o ensino à distância traz enormes desafios ao ensino superior, nomeadamente, a flexibilização dos horários, a gravação das aulas para que os estudantes possam ter acesso aos conteúdos noutros momentos e uma maior oportunidade de flexibilidade de controlo da frequência dos estudantes no acesso às plataformas educativas. Estes autores reiteram ainda que a efetividade do ensino à distância nesta era pandémica requer planeamento, organização, tecnologias da informação e comunicação disponíveis para docentes e estudantes. Ao analisar as condições para a concretização do ensino à distância, verifica-se que estas são consideradas muito boas, com disponibilidade de computador ou tablet com acesso à internet, evidência reforçada com uma participação elevada nas atividades não presenciais. Todavia, com recurso a estas plataformas educativas os estudantes demonstram preocupação com a proteção da privacidade e segurança dos dados alocados nos espaços virtuais (United Nations Educational, Scientific and Cultural Organization, 2020c), com ações de esclarecimentos sobre proteção de dados.

Dotta, Jorge, Aguiar, Silveira, \& Tedesco, (2013) afirmam que as plataformas educativas permitem acesso a muita informação, mas por si só, não promovem a aprendizagem, pelo que os docentes desempenham um papel primordial pelo seu domínio da técnica e planeamento pedagógico. Santos \& Monteiro (2020) afirmam que estes processos de transição requerem um período de ajustamento o que obrigada a uma reflexão acerca da capacidade de adaptação dos estudantes, docentes e gestores. Neste sentido, apesar do papel fulcral do docente este não deve ser entendido como uma fonte exclusiva de conhecimento, pelo que o estudante deve ter um papel proativo e 
responsável pela sua formação (Castaman \& Rodrigues, 2020).

Neste sentido, os estudantes que participaram no estudo consideram que a ESSNorteCVP manteve sempre uma atitude preditiva e adequada à evolução da pandemia pelo que frequentemente enaltecem todo o trabalho desenvolvido pela escola, com elevados níveis de satisfação pelas medidas definidas no Plano de Contingência. A adoção de medidas de confinamento social, com a suspensão das atividades presenciais na escola, restrições à movimentação de pessoas, distanciamento social e consciencialização das populações para as medidas de proteção individual, constante do plano de contingência, foram aceites e valorizadas pelos estudantes, representando uma forte adesão as atividades não presenciais, permitindo simultaneamente a interrupção da transmissão local da infeção (Cheng et al., 2020; Zhang et al., 2020). Das medidas adotadas no plano de contingência COVID-19 que mais contribuíram para reduzir o impacte do COVID-19 no processo de ensino foram a rápida adaptação da escola na criação de ambientes favoráveis ao ensino à distância síncrono com recurso a plataformas educativas; e o acompanhamento e disponibilidade constante dos docentes. Estes resultados sugerem que a ESSNorteCVP superou os desafios que a mudança de cenário de lecionação criou em termos técnicos e humanos, numa adequação dos processos de aprendizagem, para o contexto virtual, num curto espaço de tempo, através da disponibilização de suporte, apoio e formação a todos os intervenientes, condições estas que diversos contextos de ensino superior estão com dificuldades de adequação
(United Nations Educational, Scientific and Cultural Organization, 2020b).

Os estudantes reforçam a sua satisfação com a informação fornecida pela ESSNorteCVP perante o cenário atual de transmissão da infeção por COVID19 , apesar de referirem que poder-se-ia otimizar os fluxos de comunicação bidireccionalmente, através da criação de comunidades e melhoria da conexão entre as pessoas (United Nations Educational, Scientific and Cultural Organization, 2020c).

Contudo, verificamos uma satisfação mais baixa com o envolvimento/ação/representação da Associação Académica na implementação do plano de contingência COVID-19, na ótica dos próprios estudantes. O envolvimento da Associação Académica é fundamental na veiculação da informação pela sua elevada proximidade aos estudantes, o que torna primordial o desenvolvimento de mecanismos que promovam o envolvimento dos pares na implementação das medidas em resposta a estes cenários excecionais, em prol da melhoria da efetividade e eficácia das medidas a implementar.

Constata-se que durante o isolamento em casa, os estudantes revelaram um estado de maior stresse e ansiedade, assim como, estado de maior apatia e desânimo, pelo que o isolamento social pode ter efeitos negativos na saúde e bem-estar, podendo os seus efeitos persistir a longo prazo (Brooks et al., 2020). Evidencia-se assim a importância no reforço de soluções em resposta aos desafios psicossociais, das quais se destacam: implementação de medidas para avaliação da saúde mental e bem-estar dos estudantes e a promoção de debates sobre o desenvolvimento de competências e de mecanismos 
de adaptabilidade (United Nations Educational, Scientific and Cultural Organization, 2020c).

No conjunto das medidas que constam do plano de contingência referidas como boas práticas em minimizar o impacte nos processos de ensino, aprendizagem e avaliação, os estudantes reiteram e enaltecem as medidas globais adotadas pela Escola, salientando a relevância do envio para a comunidade académica dos estudos baseados na evidência acerca da temática COVID, a disponibilização de workshops, formação à distância síncrona, estudos de caso com doentes em ambiente virtual, principalmente para os estudantes que tiveram interrupção dos ensinos clínicos, pelas instituições de saúde, assim como, a poupança económica e de tempo, com a implementação do ensino à distância síncrono, referida pelos estudantes trabalhadores. A identificação de diversas iniciativas de boas práticas e de recursos dentro da comunidade académica são relevantes e significativas para que possam ser partilhadas à escala global (International Association of Universities, 2020). Neste sentido, foram apuradas um conjunto de sugestões que passam pela uniformização e desenvolvimento de regras de ensino a distância e respetiva monitorização do processo de aprendizagem dos estudantes (United Nations Educational, Scientific and Cultural Organization, 2020c), destacando: disponibilização de materiais de apoio à aula na plataforma Moodle ${ }^{\circledR}$; adoção de atividades mais dinâmicas nas aulas a decorrer nas plataformas educativas; à uniformização dos modelos de provas de avaliação via Moodle ${ }^{\circledR}$; à gestão da carga horária nos horários escolares e a manutenção do ensino à distância por forma a evitar nova vaga COVID.

\section{CONCLUSÃO}

As medidas adotadas no plano de contingência COVID-19 para minimizar os constrangimentos e limitações vivenciados pelos estudantes na consecução do seu percurso académico na ESSNorteCVP, foram adequadas tendo contribuído para reduzir o impacte do COVID-19 no processo de ensino, aprendizagem e avaliação dos estudantes. Constata-se níveis de elevada satisfação com a implementação do plano de contingência COVID-19, com a informação fornecida perante o cenário atual de COVID-19 e com níveis muito elevados de adequação das plataformas educativas para o ensino não presencial.

A rápida adaptação da escola na criação de ambientes favoráveis ao ensino à distância síncrono com recurso a plataformas educativas, assim como, o acompanhamento e disponibilidade constante dos docentes, foram as medidas identificadas que mais contribuíram para reduzir o impacte do COVID-19 no percurso académico dos estudantes, contantes no plano de contingência COVID-19.

É, ainda necessário, que face ao tempo de permanência das medidas definidas em plano de contingência seja alargado o período de monitorização, bem como, introduzidos outros instrumentos de recolha de dados, nomeadamente os relativos às questões psicossociais identificadas, e da garantia do processo de aprendizagem e respetiva avaliação de competências, para que os 
resultados obtidos sejam mais elucidativos do impacte do COVID-19.

\section{REFERÊNCIAS BIBLIOGRÁFICAS}

Brooks, S.K., Webster, R.K., Smith, L. E., Woodland, L., Wessely, S., Greenberg, N., \& Rubin, G.J. (2020). The psychological impact of quarantine and how to reduce it: rapid review of the evidence. The Lancet, 395, 912-920. doi: 10.1016/S0140-6736(20)30460-8

Camacho, F., Fuly, C., Santos, C., \& Menezes, F. (2020). Students in social vulnerability in distance education disciplines in times of COVID-19. Research, Society and Development, 9(7),1-12

Castaman, A.S., \& Rodrigues, R.A. (2020). Educação a Distância na crise COVID - 19: um relato de experiência. Research, Society and Development, 9,(6), DOI: http://dx.doi.org/10.33448/rsdv9i6.3699 1

Cheng, H., Jian, S., \& Liu, D. (2020). Contact Tracing Assessment of COVID-19 Transmission Dynamics in Taiwan and Risk at Different Exposure Periods Before and After Symptom Onset. JAMA Internal Medicine. doi:10.1001/jamainternmed.2020.2020

Decreto-Lei n.o 10-A/2020, de 13 de março. (2020). Diário da República n.ㅇ 52/2020, 1으 Suplemento, I Série. Presidência do Conselho de Ministros, Portugal.

Dotta, S., Jorge, C., Aguiar, É., Silveira, P. \& Tedesco, R., (2013). Abordagem dialógica para a condução de aulas síncronas em uma webconferência. In: X Congresso Brasileiro de Ensino Superior a Distância, 2013, Belém. Anais do X Congresso Brasileiro de Ensino Superior a Distância. Belém: Unirede/UFPA

Escola Superior de Saúde Norte da Cruz Vermelha Portuguesa. (2020). Plano de Contingência COVID19. Oliveira de Azeméis. Retirado de: https://www.essnortecvp.pt/pt/escola/covid-19/

International Association of Universities. (2020). Covid-19: Higher Education challenges and responses. Retirado de: https://www.iauaiu.net/Covid-19-Higher-Education-challenges-andresponses
Santos, J.V.B., \& Monteiro, J.C.S. (2020). Educação e covid-19: as tecnologias digitais mediando a aprendizagem em tempos de pandemia. Revista Encantar - Educação, Cultura e Sociedade - Bom Jesus da Lapa, 2, 01-15. DOI: http://dx.doi.org/10.46375/encantar.v2.0011

Sobral, S.R. (2020). O impacto do covid-19 na educação. Retirado de: https://observador.pt/opiniao/o-impacto-docovid-19-na-educacao/

United Nations Educational, Scientific and Cultural Organization. (2020a). COVID-19 Educational Disruption and Response. Retirado de: https://en.United Nations Educational, Scientific and Cultural Organization.org/covid19/educationresponse

United Nations Educational, Scientific and Cultural Organization. (2020b). Adverse consequences of school closures. Retirado de: https://en.United Nations Educational, Scientific and Cultural Organization.org/covid19/educationresponse/cons equences

United Nations Educational, Scientific and Cultural Organization. (2020c). COVID-19: 10 Recommendations to plan distance learning solutions. Retirado de: https://en.United Nations Educational, Scientific and Cultural Organization.org/news/covid-19-10recommendations-plan-distance-learning-solutions

World Health Organization. (2020). Coronavirus disease 2019 (COVID-19) Situation Report - 51. Retirado de: https://www.who.int/docs/defaultsource/coronaviruse/situation-reports/20200311sitrep-51-covid-19.pdf?sfvrsn=1ba62e57_10

Zhang, J., Litvinova, M., Wang, W., Wang, Y., Deng, X., \& Chen, X.... Yut, H. (2020). Evolving epidemiology and transmission dynamics of coronavirus disease 2019 outside Hubei province, China: a descriptive and modelling study. The Lancet Infectious Diseases. doi: 10.1016/S1473-3099(20)30230-9. 